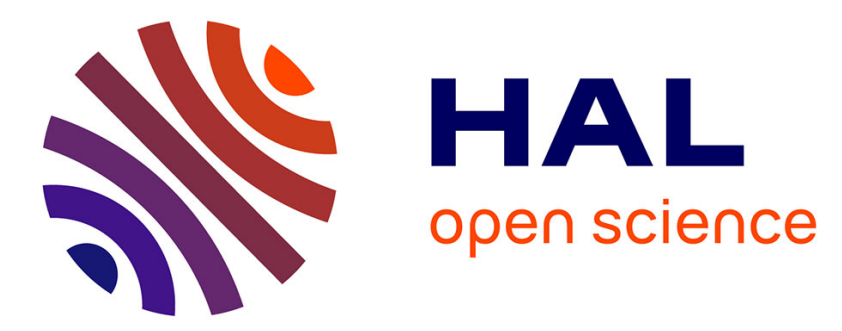

\title{
Design strategies to simplify and miniaturize imaging systems
}

Florence de La Barrière, Guillaume Druart, Nicolas Guérineau, Jean Albert Taboury

\section{> To cite this version:}

Florence de La Barrière, Guillaume Druart, Nicolas Guérineau, Jean Albert Taboury. Design strategies to simplify and miniaturize imaging systems. Applied optics, 2011, 50 (6), pp.943-951. 10.1364/AO.50.000943 . hal-00569977

\section{HAL Id: hal-00569977 \\ https://hal-iogs.archives-ouvertes.fr/hal-00569977}

Submitted on 25 May 2012

HAL is a multi-disciplinary open access archive for the deposit and dissemination of scientific research documents, whether they are published or not. The documents may come from teaching and research institutions in France or abroad, or from public or private research centers.
L'archive ouverte pluridisciplinaire HAL, est destinée au dépôt et à la diffusion de documents scientifiques de niveau recherche, publiés ou non, émanant des établissements d'enseignement et de recherche français ou étrangers, des laboratoires publics ou privés. 


\title{
Design strategies to simplify and miniaturize imaging systems
}

\author{
Florence de la Barrière, ${ }^{1, \star}$ Guillaume Druart, ${ }^{1}$ Nicolas Guérineau, ${ }^{1}$ and Jean Taboury ${ }^{2}$ \\ ${ }^{1}$ Office National d'Etudes et de Recherches Aérospatiales, Chemin de la Hunière, 91761 Palaiseau cedex, France \\ 'Institut d'Optique, Campus Polytechnique RD 128, 91127 Palaiseau cedex, France \\ ${ }^{*}$ Corresponding author: florence.de_la_barriere@onera.fr
}

Received 17 September 2010; revised 9 November 2010; accepted 9 November 2010; posted 11 November 2010 (Doc. ID 135346); published 17 February 2011

\begin{abstract}
We present the range of optical architectures for imaging systems based on a single optical component, an aperture stop, and a detector. Thanks to the formalism of third-order Seidel aberrations, several strategies of simplification and miniaturization of optical systems are examined. Figures of merit are also introduced to assess the basic optical properties and performance capabilities of such systems; by this way, we show the necessary trade-off between simplicity, miniaturization, and optical performance. (C) 2011 Optical Society of America

OCIS codes: $\quad 110.3925,220.1010,220.4830,110.4190$.
\end{abstract}

\section{Introduction}

There is currently a need for miniaturized and cheap imaging systems for both military and civilian applications. To reduce their size and their mass, imaging systems have to be as simple as possible, which means that they have to involve a minimal number of optical elements. In this paper, the simplest system is defined as a system which is composed of only three elements: a single optical component, an aperture stop, and a detector. These elements can be complex if needed. For example, optics can involve aspheric surfaces or diffractive optical elements, or can be a microlens array with a complex shape. The detector can also be curved. Indeed, a curved image surface would provide a way to lower the number of optical elements and to reduce the amount of offaxis aberrations [1], since all rays would fall quite perpendicularly to the surface of the detector.

The design of such simple systems has been widely addressed in many papers over the past decades. We can quote, for example, systems involving curved detectors [1], multichannel systems inspired by the compound eyes of insects [2-7], multichannel sys-

0003-6935/11/060943-09\$15.00/0

(C) 2011 Optical Society of America tems based on the TOMBO principle [8], lenseless imagery [9-17], and folded imaging systems [18,19]. Therefore, choosing the suitable system for a given application among all these concepts is often not obvious for an optical designer. The objective of this paper is to give design rules for simplified and miniaturized systems.

Some papers $[20,21]$ aim at evaluating the impact of the breakthrough with standard complex systems on image quality: in [20], scaling rules for optical systems are described and evaluated in terms of image quality through the introduction of the space-bandwidth product parameter. Reference [21] gives a new merit function which takes into account the field of view, the angular resolution, the sensitivity, and the volume of the optical system in a single equation. It compares the quality of two types of systems, a multichannel system and a folded annular system, to a nominal "classic" optical system, by evaluating the performance metric for each system. In this paper, we extend the classification of existing simple and miniaturized systems which can be found in literature thanks to the study of third-order Seidel aberrations.

Section 2 recalls the formalism of third-order Seidel aberrations, which aims at giving a general expression of the maximal amount of fourth-order wave aberration at the edge of the exit pupil and 
for a maximal field of view. This equation can be expressed as a function of the focal length, the field of view, and the $f$-number of the optical system, as well as the refractive index of the material, the bending of the lens, and the position of the aperture stop in the particular case of a single lens. From this equation, it turns out that different strategies can be lead to simplify an optical system. Section 3 is a review of these strategies, leading to the development of optical systems based on a single component, an aperture stop, and a detector. Through the introduction of different figures of merit, we evaluate the impact of using a single optical component on image quality, and several solutions are proposed to overcome limitations and to maintain a good optical quality.

\section{Definition of the Maximal Wave Aberration}

\section{A. Definition of the Wave Aberration}

We consider a rotationally symmetrical optical system. If this optical system is limited by the diffraction, its exit pupil function is described by a function $p_{s_{0}}(r, \varphi)$, which is the geometrical image of the aperture stop of the system [22]. The parameters $r$ and $\varphi$ are the coordinates in the exit pupil plane (see Fig. 1). The function $p_{s_{0}}(r, \varphi)$ is equal to 0 outside the pupil and to 1 inside the pupil if the illumination of the pupil is uniform.

In the general case, the optical system may not be limited by the diffraction because of aberrations; therefore, the exit pupil function $p_{s}\left(r, \varphi, r^{\prime}\right)$ is modified in the following way [23]:

$$
p_{s}\left(r, \varphi, r^{\prime}\right)=p_{s_{0}}(r, \varphi) \exp \left[i \frac{2 \Pi}{\lambda} W\left(r, \varphi, r^{\prime}\right)\right] .
$$

The function $p_{s}\left(r, \varphi, r^{\prime}\right)$ is referred to as the generalized pupil function. The parameter $r^{\prime}$ is the coordinate in the image plane (see Fig. 1).
The function $W\left(r, \varphi, r^{\prime}\right)$ is called the wave aberration. It is defined as the optical path difference between a reference sphere, which is centered on the Gaussian image of the object point, and the real wave surface at the exit pupil of the optical system.

The power series expansion of $W$ can be written in the following form [24]:

$$
W=W^{(0)}+W^{(4)}+W^{(6)}+W^{(8)}+\ldots .
$$

The general expression for the fourth-order wave aberrations is given by the following equation [25]:

$$
\begin{aligned}
W^{(4)}\left(r, \varphi, r^{\prime}\right)= & -\frac{1}{4} B r^{4}+F r^{3} r^{\prime} \cos \varphi-C r^{2} r^{\prime 2} \cos ^{2} \varphi \\
& -\frac{1}{2} D r^{2} r^{\prime 2}+E r r^{\prime 3} \cos \varphi,
\end{aligned}
$$

where $B, F, C, D$, and $E$ are coefficients. These five terms stand for third-order Seidel aberrations, respectively, spherical aberration, coma, astigmatism, field of curvature, and distortion.

B. Expressions of the Coefficients B, F, C, D, and E in the Case of a Thin Lens

We can give simplified expressions for the coefficients $B, F, C, D$, and $E$ if we consider an optical system made of a single thin lens, for which the object plane is at infinity (see Fig. 2). The thin lens is described by three parameters: the curvatures of the two refractive surfaces, $C_{1}$ and $C_{2}$, and the refractive index of the lens material $n$. Let us introduce the optical power $P$ and the bending $\gamma$ of the lens, which take into account the three parameters of interest. Their expressions are, respectively:

$$
P=\frac{1}{f}=(n-1)\left(C_{1}-C_{2}\right),
$$

$$
\gamma=\frac{1}{2}\left(C_{1}+C_{2}\right)
$$

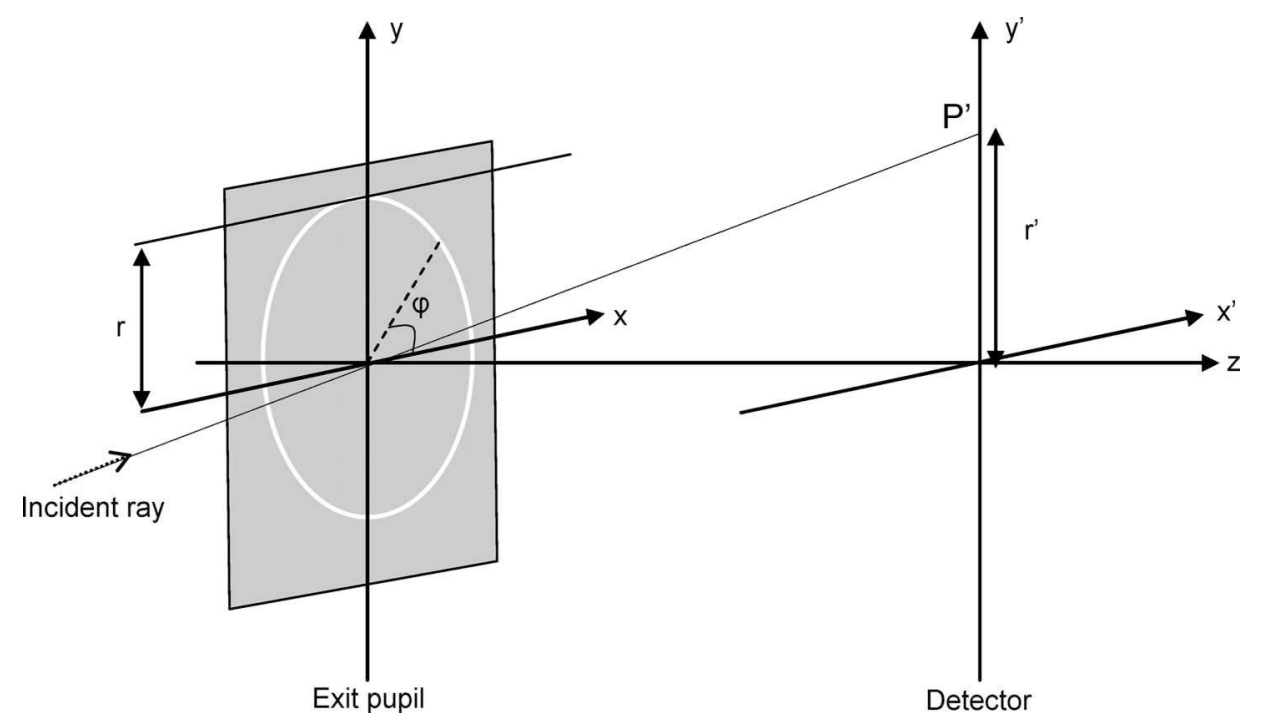

Fig. 1. Illustration of the pupil coordinates $r$ and $\varphi$ and of the image plane coordinate $r^{\prime}$. 


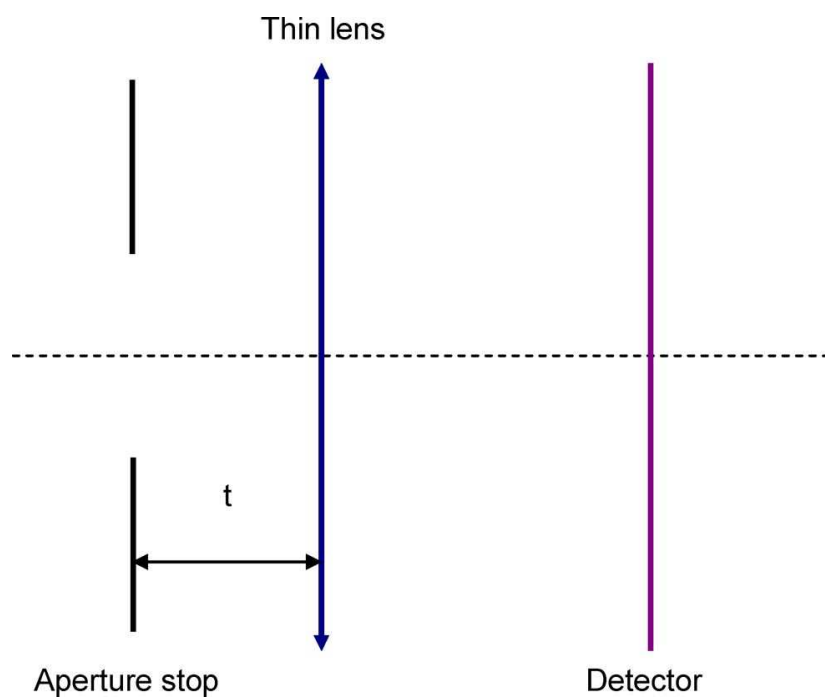

Fig. 2. (Color online) Illustration of a thin lens, an aperture stop (which is the entrance pupil), and a detector. $t$ is the distance between the entrance pupil and the lens.

The coefficients $B, F, C, D$, and $E$ can be expressed as functions of the refractive index $n$, the optical power $P$, the bending $\gamma$, and the distance $t$ between the entrance pupil and the lens [26]:

$$
\begin{gathered}
B=U, \\
F=-t U+V \\
C=t^{2} U-2 t V+\frac{P}{2} \\
D=t^{2} U-2 t V+\frac{n+1}{2 n} P, \\
E=-t^{3} U+3 t^{2} V-t \frac{3 n+1}{2 n} P,
\end{gathered}
$$

where

$$
\begin{aligned}
U= & \frac{\beta}{2}+\frac{n(4 n-1)}{8(n-1)^{2}(n+2)} P^{3} \\
+ & \frac{P}{2 n(n+2)}[(n+2) \gamma-(n+1) P]^{2}, \\
& V=\frac{P}{2 n}\left[(n+1) \gamma-\left(n+\frac{1}{2}\right) P\right] .
\end{aligned}
$$

In the expression of $U, \beta$ stands for the aspheric profiles of the surfaces of the lens: $\beta=(n-1)\left(b_{1} C_{1}^{3}-\right.$ $b_{2} C_{2}^{3}$ ), where $b_{1}$ and $b_{2}$ are the conic constants of the two surfaces of the lens.

\section{Expression of the Maximal Wave Aberration}

The number of optical elements which are needed to correct the aberrations of an optical system is linked to the amount of wave aberrations which is present in the exit pupil plane of the optical system. The maximum wave aberration which can be tolerated at the edge of the exit pupil for a maximal field angle is of particular interest.

We want the maximal field angle rays to intercept the image plane at the edge of the detector, which is supposed to be square sized, with a length $t_{\text {det }}$ in each direction. Therefore, the maximal value of $r^{\prime}$ (coordinate in the image plane) is $r_{\text {max }}^{\prime}=t_{\text {det }} / 2$. At the edge of the exit pupil, the maximal value of $r$ is $r_{\max }=$ $\phi_{s} / 2$, where $\phi_{s}$ is the diameter of the exit pupil. Under paraxial conditions, $r_{\max }^{\prime}$ and $r_{\max }$ can be expressed by the following relations: $r_{\max }^{\prime}=f \mathrm{FOV} / 2$ and $r_{\max }=f /(2 \#)$, where FOV is the field of view of the system and \# its $f$-number $\left(\#=f / \phi_{s}\right)$. Then, the maximal fourth-order wave aberration $W^{(4)}$ can be expressed as a function of $f, \mathrm{FOV}$, and \#:

$$
\begin{aligned}
W_{\max }^{(4)}(f, \#, \mathrm{FOV})= & -\frac{B}{2^{6}} \frac{f^{4}}{\#^{4}}+\frac{F}{2^{4}} \mathrm{FOV} \frac{f^{4}}{\#^{3}}-\frac{C}{2^{4}} \mathrm{FOV}^{2} \frac{f^{4}}{\#^{2}} \\
& -\frac{D}{2^{5}} \mathrm{FOV}^{2} \frac{f^{4}}{\#^{2}}+\frac{E}{2^{4}} \mathrm{FOV}^{3} \frac{f^{4}}{\#} .
\end{aligned}
$$

$W_{\max }^{(4)}$ also depends on the refractive index $n$, the bending $\gamma$, and the position $t$ of the pupil (these dependences are contained in the terms $B, F, C, D$, and $E$ ). To make things clearer, we give a simplified expression of $W_{\max }^{(4)}$ as a function of $n, \gamma, f$, \#, and FOV in the case where the aperture stop is in the plane of the thin lens (thus, $t=0$ ):

$$
\begin{aligned}
W_{\max }^{(4)}(n, \gamma, f, \#, \mathrm{FOV})= & -\frac{1}{2^{9}} \frac{n(4 n-1)}{(n-1)^{2}(n+2)} \frac{f}{\#^{4}} \\
& -\frac{1}{2^{7}(n+2)} \frac{f^{3}}{\#^{4}}[(n+2) \gamma \\
& \left.-\frac{1}{f}(n+1)\right]^{2}+\frac{n+1}{2^{5} n} \gamma f^{3} \frac{\mathrm{FOV}}{\#^{3}} \\
& -\frac{2 n+1}{2^{6} n} f^{2} \frac{\mathrm{FOV}}{\#^{3}}-\frac{1}{2^{5}} f^{3} \frac{\mathrm{FOV}^{2}}{\#^{2}} \\
& -\frac{1}{2^{6}} \frac{n+1}{n} f^{3} \frac{\mathrm{FOV}^{2}}{\#^{2}} .
\end{aligned}
$$

Depending on which parameter is chosen to minimize $W_{\max }^{(4)}$ (either $n, \gamma, f$, \#, or FOV, and in the general case $t$ ), a different optical architecture is obtained.

\section{Influence of Reducing the Maximal Wave Aberration on Traditional Figures of Merit}

Usually, the Rayleigh criterion is used to evaluate the maximal amount of aberration which can be tolerated in the optical system. The Rayleigh criterion postulates that the maximal wave aberration amount which can be tolerated at the edge of the exit pupil is equal to $\lambda / 4$ [27]. Therefore, if $W_{\max }^{(4)}$ remains inferior to $\lambda / 4$, the optical system is limited by the diffraction, and traditional figures of merit can be used to evaluate the impact of the simplification 
and miniaturization of optical systems in reference to a nominal "classic" optical system (for example, a single thin lens). These traditional figures of merit are recalled in the following subsections.

\section{Angular Resolution and Number of Resolved Points}

The angular resolution instantaneous field of view quantifies the ability of the optical system to distinguish small details [28]. It is linked to the maximum spatial frequency $\nu_{\max }$ that can be resolved by the optical system. $\nu_{\max }$ can be calculated as the ratio between the blur caused by the optical system and its focal length. Two main factors contribute to the blur caused by the optical system (the diffraction spot size and the geometrical spot size), and it is commonplace to combine these effects by calculating the square root of their quadratic sum [20]. As we have just mentioned at the beginning of this section, we consider that the optical system is limited by the diffraction, so that the diffraction spot size becomes preponderant in relation to the geometrical spot size of the optical system. Thus, IFOV can be expressed by the following equation:

$$
\mathrm{IFOV}=\frac{\lambda \#}{f} .
$$

The number of resolved points is defined as follows:

$$
N_{b}=\left(\frac{\mathrm{FOV}}{\mathrm{IFOV}}\right)^{2}
$$

By introducing Eq. (15) into Eq. (16), the number of resolved points can be expressed in the following way as a function of $f, \mathrm{FOV}$, and \#:

$$
N_{b}=\frac{f^{2} \mathrm{FOV}^{2}}{\lambda^{2} \#^{2}} .
$$

$N_{b}$ does not depend on $n, \gamma$, and $t$; therefore, it is suitable to play on these parameters to reduce the maximal amount of aberrations. However, like $W_{\max }^{(4)}, N_{b}$ is an increasing function of $f$ and FOV, and it is a decreasing function of \#. Thus, playing on $f, \mathrm{FOV}$, and \# to minimize $W_{\max }^{(4)}$ results in a decrease of the number of resolved points. A trade-off between the simplification of an optical system and the number of resolved points is sometimes necessary and it can be summarized in the following way: "Bigger is better but small is best" [29]. Having less resolved points is the price to pay for widespread optical systems in simple and cheap applications.

\section{2. Étendue}

The étendue is linked to the ability for an optical system of collecting light. If we want to image a scene, the étendue $G$ is inversely proportional to the square of the $f$-number for an object at infinity:

$$
G=\frac{\Pi t_{\mathrm{pix}}^{2}}{4 \#^{2}} .
$$

In this paper, we consider that the size of the pixel is adapted to the radius of the Airy pattern, thus, $G$ no longer depends on the $f$-number:

$$
G=1.17 \lambda^{2} .
$$

In this case, the étendue remains constant while miniaturizing an optical system which is limited by the diffraction.

\section{Strategies for the Simplification and the Miniaturization of Optical Systems}

A. Playing on the Refractive Index, the Bending of the Lens, and the Position of the Pupil

Simple considerations based on Eqs. (6)-(10) can be carried out. We first consider that the entrance pupil is in the plane of the lens $(t=0)$. The bending $\gamma$ appears both in the spherical aberration term (dependence with $\gamma^{2}$ ) and in the coma term (dependence with $\gamma$ ). Therefore, two choices are possible for the value of $\gamma$. The first one consists of minimizing the spherical aberration, so that the suitable value of $\gamma$ is given by

$$
\gamma_{\min }=\frac{1}{f} \frac{n+1}{n+2} .
$$

The second one consists of canceling the coma aberration; in this case, $\gamma$ has to be chosen according to the following equation:

$$
\gamma_{(\mathrm{coma}=0)}=\frac{1}{f} \frac{2 n+1}{2(n+1)} .
$$

If we choose the bending according to Eq. (21), it is possible to correct simultaneously spherical aberration and coma just by giving aspheric profiles to the surfaces of the lens $(\beta \neq 0)$. If the pupil remains in the plane of the lens, the astigmatism term cannot be canceled; however, it can be reduced just by moving away the pupil from the lens $(t \neq 0)$, which results in introducing a little amount of coma, and distortion. Field curvature can be reduced by choosing a high refractive index of the material. This approach has been used to design a simple system working in the infrared spectral bandwidth. This simple system is only composed of an aperture stop used as the entrance pupil of the system, a Silicon meniscus, and a planar detector [30].

If playing on the refractive index, the bending, and the position of the pupil is not sufficient to provide a good optical quality, the traditional approach consists of increasing the number of optical surfaces, which often leads to increasing the number of optical components. However, a method which increases the number of optical surfaces while using a single optical component has already been proposed: the design 
is based on a folded imaging system which reflects the optical path multiple times with concentric reflectors $[18,19]$. The limitation of such folded systems is that the field of view is narrowed as the number of reflective surfaces increases. However, it is possible to maintain a constant étendue by keeping the area of the pupil constant with respect to a classic nonobscured system. Other approaches which consist of playing on $f$, FOV, and \# result in decreasing $W_{\max }^{(4)}$ without increasing the number of optical surfaces. These approaches are described in the following subsections.

\section{B. Increasing the f-Number \#: Lenseless Imagery}

By choosing a high $f$-number for the optical system, no focusing element is required to image a scene. This corresponds to the simplest optical systems. For example, the pinhole camera is only composed of an aperture stop with a low-diameter value $[9,10]$.

We note $M$ the scaling factor, that is to say that $\#_{2}=M \#_{1}$, where $\#_{1}$ is the $f$-number of a nominal "classic" system. IFOV is thus affected in the following way: $\mathrm{IFOV}_{2}=M \mathrm{IFOV}_{1}$, and the number of resolved points scales with $1 / M^{2}: N_{b 2}=N_{b 1} / M^{2}$.

Note that the off-axis étendue can be improved if a curved detector is associated with the pinhole [9].

The low angular resolution IFOV provided by a pinhole camera can be improved by using other lenseless imaging systems, such as coded apertures. In the case of coded apertures, the scene is no longer imaged by a single pinhole, but by many pinholes properly arranged [11-13]. Other lenseless imaging concepts involve a circular diffraction grating. It has been shown that circular diffraction gratings, which belong to the class of self-imaging objects, concentrate the light along a focal line and therefore have an imaging property $[14,15]$. Continuously self-imaging gratings (CSIGs), which are also self-imaging objects, have even been studied by using the formalism of thirdorder Seidel aberrations [31]. This formalism enables one to compare the performance of CSIGs with the performance of classical lenses. Alternatives to circular diffraction gratings, such as diffractive optics or holographic axilens, have also been studied for imaging properties $[16,17]$. One of the advantages of all lenseless imaging systems is their great depth of focus.

\section{Decreasing the FOV: Multichannel Insect's Eyes}

Another way to reduce the maximal amount of fourth-order wave aberrations is to decrease the field of view of the optical system $\left(\mathrm{FOV}_{2}=\mathrm{FOV}_{1} / M\right)$. Although IFOV remains the same in reference to a nominal classic optical system, the number of resolved points decreases as $1 / M^{2}\left(N_{b 2}=N_{b 1} / M^{2}\right)$.

However, if the solid angle is carefully divided into $M^{2}$ optical channels (all the channels having tilted optical axes), the field of view, and thus the number of resolved points, remains constant in reference to a nominal classic optical system. This approach is directly inspired by the compound eyes of insects [2-7].
Therefore, the multichannel approach reduces the field of view of each channel $\mathrm{FOV}_{e}$ while keeping $N_{b}$ constant. However, great care must be taken because the field of view of each channel $\left(\mathrm{FOV}_{e}\right)$ depends on the $f$-number of a single channel and is limited by the geometrical design of the optical system, which changes between curved and planar configurations. Based on the notations of Fig. 3(a) for a curved system and of Fig. 3(b) for a planar system, it turns out that the $f$-number is linked to the field of view $\mathrm{FOV}_{e}$ of a single optical channel through the following equations (provided the angles $\mathrm{FOV}_{e}$ and $\theta$ are small):

$$
\#_{\text {curved }}=\frac{1}{\theta+\mathrm{FOV}_{e}},
$$

for a curved system, where $\theta$ is the angle between the axes of successive channels of the optical system, and

$$
\#_{\text {planar }}=\frac{1}{\mathrm{FOV}_{e}},
$$

for a planar system. In the case illustrated in Fig. 3(b), each channel has the same $\mathrm{FOV}_{e}$, and the overall FOV will be increased by adding a beam deflector at the top of the system, so that the optical axes of the channels are tilted in respect to each other.

As the angles $\mathrm{FOV}_{e}$ and $\theta$ are small, they can be expressed in the following way:

$$
\begin{gathered}
\mathrm{FOV}_{e} \approx \frac{t_{\mathrm{det}}}{f}, \\
\theta \approx \frac{t_{\mathrm{det}}}{e} .
\end{gathered}
$$

A simple ray tracing through the system shows that if $\theta>\mathrm{FOV}_{e}$ (that is to say $e<f$ ), spatial lacunarities appear in the image because some areas of the scene are not imaged by the system. In general, this case is not suitable for practical imaging applications. If $\theta=$ FOV $_{e}$ (that is to say $e=f$ ), which is illustrated in Fig. 3(a), the scene is perfectly tiled between the different optical channels, and the overall field of view $\mathrm{FOV}$ of the system is given by $\mathrm{FOV}=M \mathrm{FOV}$, where $M$ stands for the number of channels in one direction. If $\theta<\mathrm{FOV}_{e}$ (that is to say $e>f$ ), overlap areas are provided between adjacent channels, which can help to retrieve a single image from the collection of subimages provided by the multichannel system.

The $f$-number of a single optical channel is greater for a planar system than for a curved one: therefore, it is more convenient to design a curved system in order to work with a lower value of \#. The minimal $f$ number for a curved system can be obtained by tiling the scene into the different optical channels without providing any overlap area between the channels. In this case, the $f$-number of the curved system is given by the following expression: 


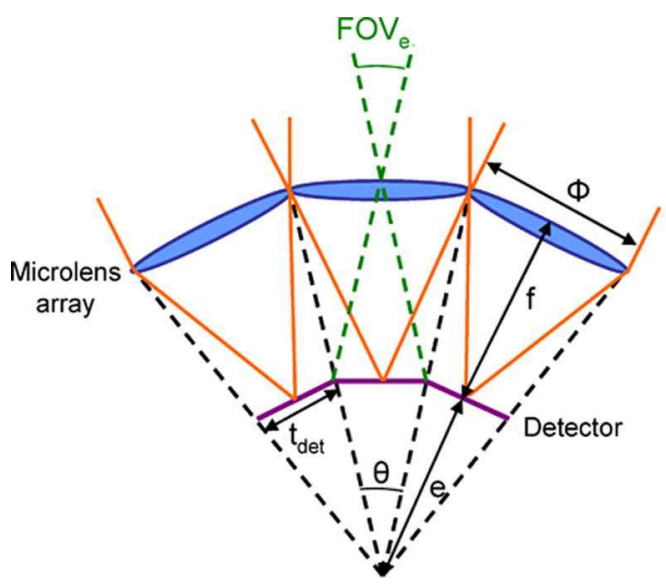

(a)

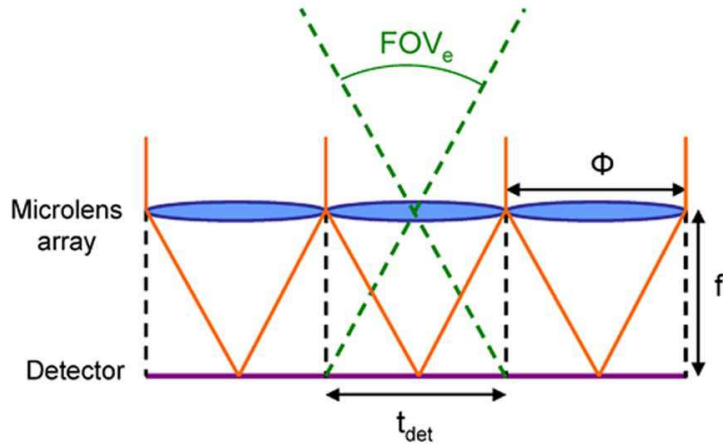

(b)

Fig. 3. (Color online) Illustration of a multichannel optical system (a) with curved components, (b) with planar components.

$$
\#_{\text {curved }}=\frac{1}{2 \mathrm{FOV}_{e}}
$$

so that

$$
\#_{\text {curved }}=\frac{\#_{\text {planar }}}{2} \text {. }
$$

That is why a curved microlens array associated with a curved detector would convey the best results [32] [this configuration is directly based on the apposition compound eyes of the fly (see Fig. 4)].

From a practical point of view, the field of view $\mathrm{FOV}_{e}$ of one channel is chosen so that the maximum amount of fourth-order wave aberration $W_{\max }^{(4)}$ fulfils the Rayleigh criterion $\left(W_{\max }^{(4)} \leq \lambda / 4\right)$. Then, the optimal number of channels $N_{\mathrm{ch}}$ is linked to the desired overall FOV of the system through the following relation: $N_{\mathrm{ch}}=\mathrm{FOV} / \mathrm{FOV}_{e}$.

However, current state-of-the-art technology implies the common use of planar components, such as planar detectors and planar microlens arrays. Thus, the design becomes more complex, because it has to address two main difficulties (tilting the optical axis of each channel by using a planar component and suppressing cross talk between adjacent channels). Other elements must be added to the design, such as a beam deflector $[\underline{7}, \underline{8}, \underline{33}]$ or thin and long opaque walls between the microlens array and the detector $[\underline{4}, \underline{8}]$.

\section{Decreasing the Focal Length $f$ : The TOMBO Principle}

The final way to decrease $W_{\max }^{(4)}$ consists of decreasing the focal length $f$ of the system. However, according to Eqs. (15) and (17), miniaturizing an optical system by decreasing its focal length while maintaining a constant FOV and a constant $f$-number results in a decrease of the angular resolution IFOV and of the number of resolved points $N_{b}$ [20]: if $f_{2}=f_{1} / M$, then $\mathrm{IFOV}_{2}=M \mathrm{IFOV}_{1}$ and $N_{b 2}=\bar{N}_{b 1} / M^{2}$. This is illustrated in Fig. 5(a). Several solutions could be used to compensate for this loss of resolved points [34]. One of these solutions is to design a multichannel sys- tem by replicating a miniaturized imaging system [see Fig. 5(b)], with each channel providing nonredundant information. Each subimage is undersampled; that is why an image processing method has to be applied to the set of undersampled subimages to obtain a final image with an enhanced angular resolution. According to the sampling theorem of Papoulis [35], if each subimage is undersampled by a factor $M^{2}$, a single image can be retrieved from a collection of $M^{2}$ nonredundant subimages, avoiding a loss of information. This approach relies on image reconstruction algorithms which are based on nonredundant information provided by the optical channels. To obtain this nonredundancy, practical challenges have to be achieved, such as introducing subpixel shifts between the subimages. These subpixel shifts can be obtained either by choosing a microlens array pitch which is not a multiple of the size of the pixel, or by tilting the optical system in relation to the axes of the detector [34]. If the relative subimage shifts are determined once and for all by calibrating the camera, the image reconstruction becomes impervious to changes in subimage content, contrast, sharpness, and noise [36].

The Nyquist frequency of the detector is given by $f_{N y}=1 /\left(2 p_{s}\right)$, where $p_{s}$ is the sampling pitch of the detector. The size of the pixels $t_{\text {pix }}$ is inferior to the sampling pitch $\left(t_{\text {pix }} \leq p_{s}\right)$, and we define the fill factor $F$ of the pixels by the following equation:

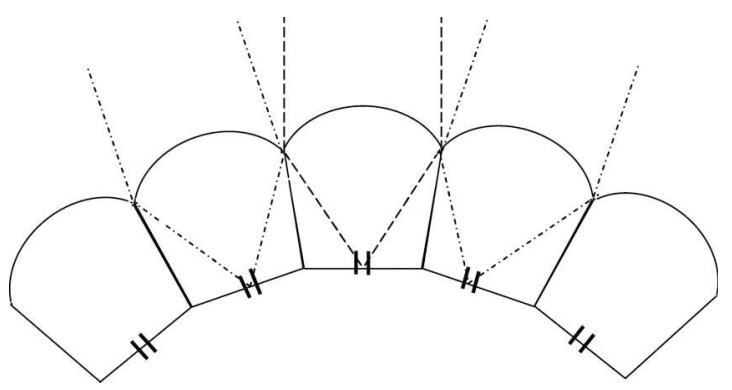

Fig. 4. Illustration of an apposition compound eye (which corresponds to the eye of the fly), with a curved microlens array and a curved retina in a convex shape (see []]). 

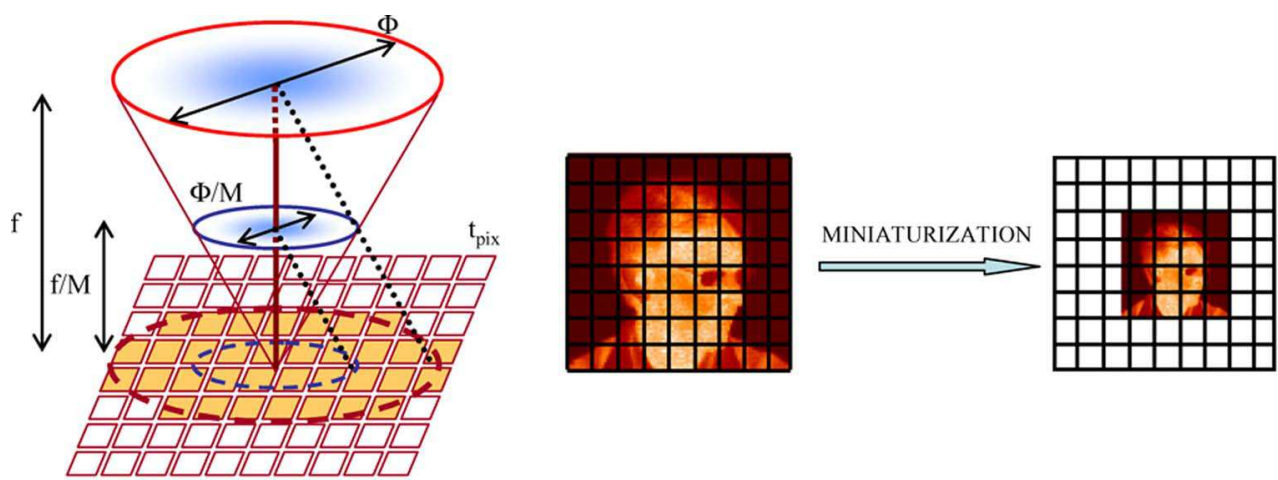

(a)
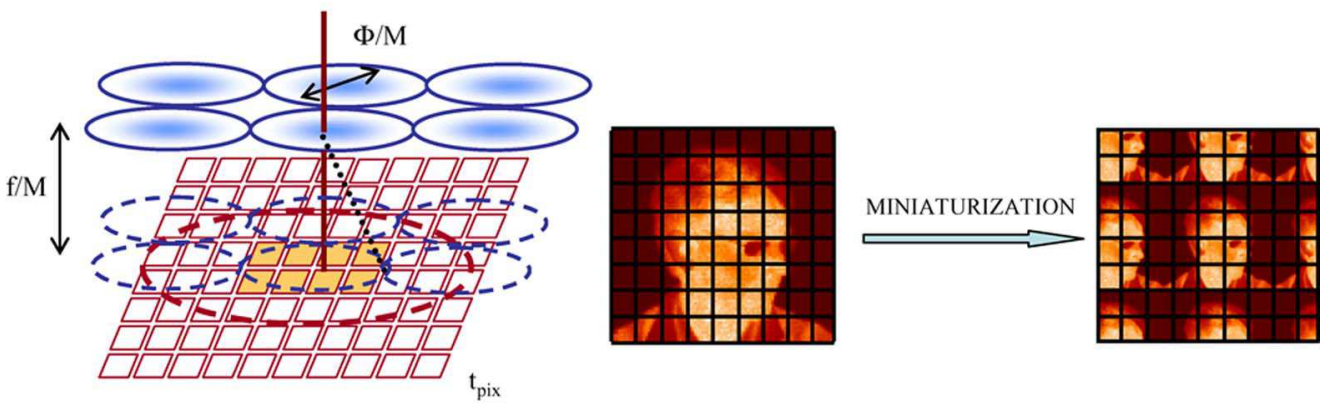

(b)

Fig. 5. (Color online) (a) Illustration of the miniaturization of an optical system by decreasing its focal length $f$, while maintaining a constant $f$-number and a constant field of view, and illustration of the decrease of the number of resolved points. (b) Method to compensate for the loss of resolved points by replicating an optical miniaturized system.

$$
F=\left(\frac{t_{\text {pix }}}{p_{s}}\right)^{2}
$$

Thus, $p_{s}=t_{\text {pix }}$ corresponds to the case where the fill factor of the pixels is equal to 1 . We can only expect to retrieve frequencies until the cutoff frequency of the

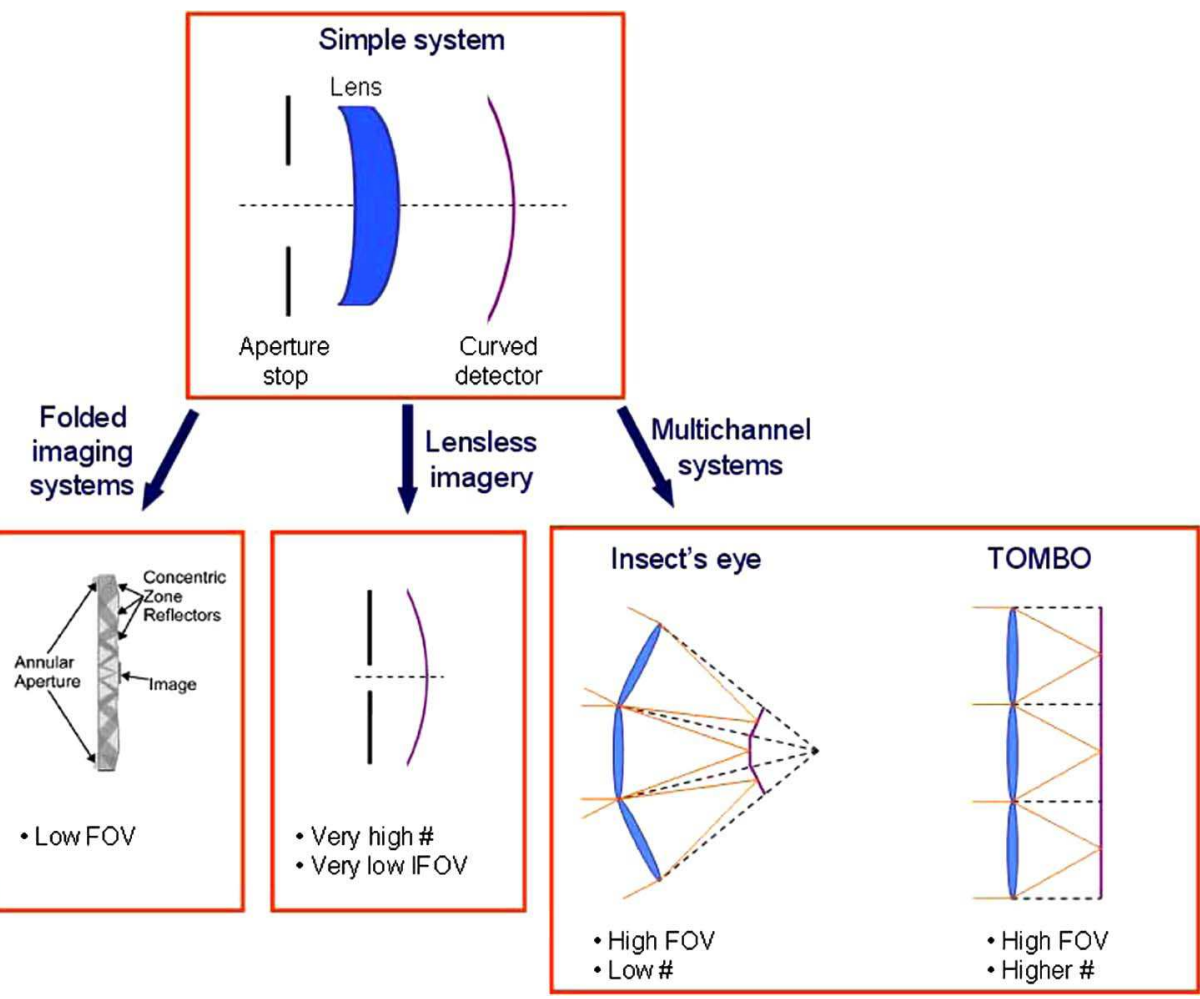

Fig. 6. (Color online) Illustration of the different strategies used to design a simple imaging system with a minimal number of optical elements. 
pixel $1 / t_{\text {pix }}$. Therefore, if the fill factor of the pixels is equal to 1 , the Nyquist frequency is $f_{N y}=1 /\left(2 t_{\mathrm{pix}}\right)$, and a system based on the TOMBO principle with two nonredundant channels in each direction would enable one to retrieve frequencies until twice the Nyquist frequency. If we want to retrieve frequencies higher than twice the Nyquist frequency, the fill factor of the pixels has to be reduced in order to increase the cutoff frequency of the pixels, and the number of required nonredundant channels has to be increased. The optimal number of nonredundant channels $N_{\mathrm{ch}}$ is given by the following equation:

$$
N_{\mathrm{ch}}=\frac{2}{\sqrt{F}} .
$$

For example, if the fill factor of the pixels is equal to $F=0.25$ (that is to say $t_{\text {pix }}=p_{s} / 2$ ), the optimal number of nonredundant channels is $N_{\mathrm{ch}}=4$. The problem which arises in image reconstruction is that the contrast of high frequencies (up to the cutoff frequency of the pixel) is very low and even equal to 0 at the cutoff frequency of the pixel.

\section{Conclusion}

In this paper, we have described different strategies to design a simple imaging system based on a single optical component. These strategies are summarized graphically in Fig. 6. They are all deduced from the theory of third-order Seidel aberrations. We can see that playing on different parameters (the index of refraction $n$, the bending $\gamma$, the position of the pupil $t$, the focal length $f$, the field of view, and the $f$-number) reduces the amount of fourth-order wave aberration of the system. It is more convenient to play on $n, \gamma$, and $t$ since these parameters do not affect the resolution of the system. However, if it is not sufficient, the classic approach consists of increasing the number of optical elements in order to increase the number of optical surfaces. Nevertheless, we have shown that other approaches keep on using a single optical component. Annular folded systems increase the number of optical surfaces while keeping a single optical component. Playing on \#, FOV, and $f$ enables the design of simple or miniaturized systems, but it results in a decrease of the number of resolved points. The interest of multichannel optical systems is that they can be very compact while maintaining a satisfying number of resolved points. From a practical point of view, although multichannel systems rely on simple optical architectures, the unique optical element which is used can be complex. Curved detectors, curved microlens arrays, and planar microlens arrays with potentially important optical power are not mature yet. Suppressing cross talk between adjacent channels is also an important issue. Then, multichannel systems have to overcome technological challenges to be widespread in a large range of applications.

This work was sponsored by the Délégation Générale pour l'Armement (DGA) of the French Ministry of Defense.

\section{References}

1. S. B. Rim, P. B. Catrysse, R. Dinyari, K. Huang, and P. Peumans, "The optical advantages of curved focal plane arrays," Opt. Express 16, 4965-4971 (2008).

2. R. Völkel, M. Eisner, and K. J. Weible, "Miniaturized imaging systems," Microelectron. Eng. 67-68, 461-472 (2003).

3. J. Duparré, P. Dannberg, P. Schreiber, A. Bräuer, and A. Tünnermann, "Artificial apposition compound eye fabricated by micro-optics technology," Appl. Opt. 43, 4303-4310 (2004).

4. J. Duparré, P. Dannberg, P. Schreiber, A. Bräuer, and A. Tünnermann, "Thin compound eye camera," Appl. Opt. 44, 2949-2956 (2005).

5. J. Duparré, P. Schreiber, A. Matthes, E. Pshenay-Severin, A. Bräuer, A. Tünnermann, R. Völkel, M. Eisner, and T. Scharf, "Microoptical telescope compound eye," Opt. Express 13, 889903 (2005)

6. J. W. Duparré, and F. C. Wipermann, "Micro-optical artificial compound eyes," Bioinsp. Biomim. 1, R1-R16 (2006).

7. G. Druart, N. Guérineau, R. Haïdar, S. Thétas, J. Taboury, S. Rommeluère, J. Primot, and M. Fendler, "Demonstration of an infrared microcamera inspired by Xenos Peckii vision," Appl. Opt. 48, 3368-3374 (2009).

8. J. Tanida, T. Kumagai, K. Yamada, S. Miyatake, K. Ishida, T. Morimoto, N. Kondou, D. Miyazaki, and Y. Ichioka, "Thin observation module by bound optics (TOMBO): Concept and experimental verification," Appl. Opt. 40, 1806-1813 (2001).

9. G. Druart, N. Guérineau, J. Taboury, S. Rommeluère, R. Haïdar, J. Primot, M. Fendler, and J. C. Cigna, "Compact infrared pinhole fisheye for wide field applications," Appl. Opt. 48, 1104-1113 (2009).

10. S. R. Wilk, "Ancient optics: Producing magnification without lenses," Opt. Photonics News 17 (4), 12-13 (2006).

11. G. Andersen and D. Tullson, "Broadband antihole photon sieve telescope," Appl. Opt. 46, 3706-3708 (2007).

12. E.E.Fenimore and T.M.Cannon, "Coded aperture imaging with uniformly redundant arrays,” Appl. Opt. 17, 337-347 (1978).

13. S. R. Gottesman and E. E. Fenimore, "New family of binary arrays for coded aperture imaging," Appl. Opt. 28, 43444352 (1989).

14. G. Druart, N. Guérineau, R. Haïdar, J. Primot, A. Kattnig, and J. Taboury, "Image formation by use of continuously selfimaging gratings and diffractive axicons," Proc. SPIE 6712, 671208 (2007).

15. G. Druart, J. Taboury, N. Guérineau, R. Haïdar, H. Sauer, A. Kattnig, and J. Primot, "Demonstration of image-zooming capability for diffractive axicons," Opt. Lett. 33, 366-368 (2008).

16. G. Mikula, A. Kolodziejczyk, M. Makowski, C. Prokopowicz, and M. Sypek, "Diffractive elements for imaging with extended depth of focus," Opt. Eng. (Bellingham, Wash.) 44, 058001 (2005).

17. N. Davidson, A. A. Friesem, and E. Hasman, "Holographic axilens: High resolution and long focal depth," Opt. Lett. 16, 523-525 (1991).

18. E. J. Tremblay, R. A. Stack, R. L. Morrison, and J. E. Ford, "Ultrathin cameras using annular folded optics," Appl. Opt. 46, 463-471 (2007).

19. E. J. Tremblay, R. A. Stack, R. L. Morrison, J. H. Karp, and J. E. Ford, "Ultrathin four-reflection imager," Appl. Opt. 48, 343354 (2009).

20. A. W. Lohmann, "Scaling laws for lens systems," Appl. Opt. 28 , 4996-4998 (1989).

21. M. W. Haney, "Performance scaling in flat imagers," Appl. Opt. 45, 2901-2910 (2006).

22. J. W. Goodman, Introduction to Fourier Optics, 3rd ed. (Roberts and Company, 2005), p. 107.

23. J. W. Goodman, Introduction to Fourier Optics, 3rd ed. (Roberts and Company, 2005), p. 146. 
24. M. Born and E. Wolf, Principles of Optics, 6th ed. (Pergamon, 1989), p. 211.

25. M. Born and E. Wolf, Principles of Optics, 6th ed. (Pergamon, 1989), p. 213.

26. M. Born and E. Wolf, Principles of Optics, 6th ed. (Pergamon, 1989), p. 228.

27. M. Born and E. Wolf, Principles of Optics, 6th ed. (Pergamon, 1989), p. 468.

28. G. Druart, N. Guérineau, R. Haïdar, M. Tauvy, S. Thétas, S. Rommeluère, J. Primot, J. Deschamps, and E. Lambert, "MULTICAM: A miniature cryogenic camera for infrared detection," Proc. SPIE 6992, 699215 (2008).

29. D. J. Brady, "Micro-optics and megapixels," Opt. Photonics News, 17(11), 24-29 (2006).

30. F. de la Barrière, G. Druart, N. Guérineau, J. Taboury, and M. Fendler, "Integration of advanced optical functions near the focal plane array: First steps towards the on-chip infrared camera," Proc. SPIE 7787, 778706 (2010).
31. G. Druart, N. Guérineau, R. Haïdar, J. Primot, P. Chavel, and J. Taboury, "Nonparaxial analysis of continuous self-imaging gratings in oblique illumination,” J. Opt. Soc. Am. A 24, 33793387 (2007).

32. N. A. Ahuja and N. K. Bose, "Design of large field-of-view high-resolution miniaturized imaging system," EURASIP J. Adv. Signal Process. 2007, 1 (2007).

33. L. C. Laycock and V. A. Handerek, "Multi-aperture imaging device for airborne platforms," Proc. SPIE 6737, 673709 (2007).

34. F. de la Barrière, G. Druart, N. Guérineau, J. Taboury, J. Primot, and J. Deschamps, "Modulation transfer function measurement of a multichannel optical system," Appl. Opt. 49, 2879-2890 (2010).

35. A. Papoulis, "Generalized sampling expansion," IEEE Trans. Circuits Syst. 24, 652-654 (1977).

36. A. V. Kanaev, J. R. Ackerman, E. F. Fleet, and D. A. Scribner, "TOMBO sensor with scene-independent superresolution processing," Opt. Lett. 32, 2855-2857 (2007). 